\title{
Retroviruses in Wild-Born Semi-Captive East African Sanctuary Chimpanzees (Pan troglodytes schweinfurthii)
}

\author{
Lawrence Mugisha ${ }^{1,2}$, Claudia Kücherer ${ }^{3}$, Heinz Ellerbrok ${ }^{3}$, Sandra Junglen ${ }^{3}, J^{\prime}$ ohn Opuda-Asibo ${ }^{4}$, \\ Olobo O. Joseph ${ }^{5}$, Georg Pauli ${ }^{3}$ and Fabian H. Leendertz ${ }^{*},{ }^{3}$
}

\author{
${ }^{I}$ Chimpanzee Sanctuary \& Wildlife Conservation Trust (CSWCT), P.O. Box, 884, Entebbe, Uganda \\ ${ }^{2}$ Makerere University, Faculty of Veterinary Medicine, Department of Wildlife and Animal Resources Management \\ (WARM), P.O. Box 7062, Kampala, Uganda \\ ${ }^{3}$ Robert Koch-Institut, (various units) Nordufer 20, D-13353 Berlin, Germany \\ ${ }^{4}$ Makerere University, Faculty of Veterinary Medicine, Department of Veterinary Public Health and Preventive \\ Medicine, P.O. Box 7062, Kampala, Uganda \\ ${ }^{5}$ College of Health Sciences, School of Biological Science, Department of Microbiology, P.O. Box 7072, Kampala, \\ Uganda
}

\begin{abstract}
Information on retroviruses infections in great apes is scarce, especially for apes kept in sanctuaries throughout Africa. To investigate the prevalence of retroviruses and possible transmission of different retroviruses originating from chimpanzees of different origin (Uganda, Congo and Rwanda), 38 wild-born captive orphan chimpanzees residing in a sanctuary on Ngamba Island were analyzed for retroviral infections. Samples from sanctuary chimpanzees were analyzed using enzyme-linked immunoassays and polymerase chain reactions (PCR). Viruses were characterized by phylogenetic analysis. All chimpanzees were negative for antibodies against Simian Immunodeficiency Virus (SIV) and Simian T-cell Leukemia Virus (STLV). However, 28/38 (73\%) chimpanzees were positive to Simian Foamy Virus (SFV) by analysis of a 425-bp DNA segment obtained by PCR using generic integrase primers homologous to highly conserved portions of the polymerase gene. Phylogenetic analysis of SFV sequences obtained in this study formed four sub clusters within the specific SFV P. t. schweinfurthii clade with significant variability among the new SFVs strains.

We provide evidence of on-going cross-transmission of SFV among chimpanzees within the sanctuary mostly likely through horizontal routes. We propose to test all chimpanzees introduced into sanctuaries for retroviral and other infections. This will help avoid the spread also of pathogenic viruses in captive populations.
\end{abstract}

Keywords: SIV, STLV, SFV, orphan chimpanzees.

\section{INTRODUCTION}

Simian Immunodeficiency Virus (SIV), Simian T-cell Leukemia Virus (STLV) and Simian Foamy Virus (SFV) are known to occur in wild populations of chimpanzees. However, the prevalence of these viruses varies significantly. SIV is found only in some specific groups of wild chimpanzees with low prevalence within the group [1, 2]. SFV in contrast was present in all wild chimpanzee groups studied. The prevalence rates ranged from $44 \%$ to $100 \%$ with several individuals infected with more than one strain $[3,4]$. An age-dependent prevalence was revealed, with older individuals showing a higher prevalence [4]. Not much is known about the prevalence of STLV in wild chimpanzees. Data from Côte d'Ivoire suggest a significantly higher prevalence among chimpanzees older than nine years with a seroprevalence of $71.4 \%$ compared to $9.7 \%$ in individuals younger than nine years [5].

*Address correspondence to this author at the Research Group Emerging Zoonoses, Robert Koch-Institut, Nordufer 20, D-13353 Berlin, Germany; Tel: +49 3018754 2592; E-mail: LeendertzF@rki.de
To understand the age distribution of animals brought to chimpanzee sanctuaries, it has to be mentioned that in order to capture one infant chimpanzee from their community in the natural habitat, poachers have to shoot three to five adults. The adults are used for commercial/domestic bushmeat trade while the babies are sold for pet trade. The latter are those ending in many cases in sanctuaries throughout Africa. Capturing of wild chimpanzees is strictly forbidden in all great ape range countries but still every year many infant chimpanzees are confiscated from illegal dealers or private owners. Between 2001 and 2005, 5 to 95 infant chimpanzees were received through confiscations per year in 19 Pan African Sanctuary Alliance (PASA) primate sanctuaries though the trend is declining in the last years (PASA, unpublished data). The age of rescued infant chimpanzees ranges from one to five years with some being rescued after staying with their illegal owners for two to three years.

Here we show investigations on the prevalence of SIV, STLV and SFV in wild-born chimpanzees of the Eastern subspecies ( $P$. $t$ t. schweinfurthii) at the Ngamba Island Chimpanzee Sanctuary (NICS), Uganda. Animals originated 
Table 1. Name, Origin, Sex and Results of Foamy Virus PCR of Chimpanzees on Ngamba Island

\begin{tabular}{|c|c|c|c|c|c|c|c|}
\hline Name & Sex & $\begin{array}{l}\text { Est. Yr of } \\
\text { Birth }\end{array}$ & $\begin{array}{c}\text { Age (yrs) } \\
\text { by } 2007\end{array}$ & Year of Arrival & Origin & $\begin{array}{l}\text { First Round } \\
\text { PCR }\end{array}$ & $\begin{array}{c}\text { Integrase } \\
\text { Nested PCR }\end{array}$ \\
\hline Kidogo & Female & 1984 & 23 & 1998 & Congo & - & - \\
\hline Sophie & Female & 1986 & 21 & 1998 & Congo & - & - \\
\hline Katie & Female & 1987 & 20 & 1998 & Congo & - & - \\
\hline Ikuru & Female & 1995 & 12 & 1999 & Congo & + & + \\
\hline Billi & Female & 1998 & 10 & 2001 & Congo & - & + \\
\hline Mukisa & Female & 1998 & 9 & 2001 & Congo & - & + \\
\hline Ndyakira & Female & 1999 & 8 & 2002 & Congo & - & - \\
\hline Nakuu & Female & 2001 & 6 & 2003 & Congo & + & + \\
\hline Peace & Female & 1986 & 21 & 1998 & Uganda & $(+)$ & $(+)$ \\
\hline Connie & Female & 1989 & 18 & 1998 & Uganda & - & + \\
\hline Bahati & Female & 1990 & 17 & 1998 & Uganda & - & - \\
\hline Natasha & Female & 1990 & 17 & 1998 & Uganda & - & + \\
\hline Becky & Female & 1991 & 16 & 1998 & Uganda & - & + \\
\hline Sally & Female & 1991 & 16 & 1998 & Uganda & - & - \\
\hline Cindy & Female & 1993 & 14 & 1998 & Uganda & - & $(+)$ \\
\hline Nkumwa & Female & 1996 & 11 & 1998 & Uganda & - & $(+)$ \\
\hline Kazahukire & Female & 1999 & 8 & 2002 & Uganda & + & + \\
\hline Kyewunyo $^{1}$ & Female & 2002 & 5 & 2002 & Uganda & - & + \\
\hline Megan & Female & 1984 & 23 & 1998 & Rwanda & - & $(+)$ \\
\hline Nagoti & Female & 1986 & 21 & 1998 & Unknown & - & + \\
\hline Nani & Female & 2001 & 6 & 2002 & Unknown & + & + \\
\hline Masiko & Male & 1984 & 23 & 1998 & Congo & + & + \\
\hline Sunday & Male & 1987 & 20 & 1998 & Congo & - & + \\
\hline Robbie & Male & 1986 & 21 & 1998 & Congo & - & + \\
\hline Mawa & Male & 1996 & 11 & 1999 & Congo & + & + \\
\hline Kalema & Male & 1996 & 11 & 1999 & Congo & - & - \\
\hline Umutama & Male & 1996 & 11 & 1999 & Congo & + & + \\
\hline Umugezi & Male & 1997 & 10 & 1999 & Congo & - & + \\
\hline Baluku & Male & 1998 & 9 & 1999 & Congo & - & + \\
\hline Asega & Male & 1998 & 9 & 2000 & Congo & - & + \\
\hline Kisembo & Male & 1999 & 8 & 2000 & Congo & - & - \\
\hline Indi & Male & 1999 & 8 & 2001 & Congo & - & $(+)$ \\
\hline Okech & Male & 2001 & 7 & 2003 & Congo & - & + \\
\hline Rambo & Male & 2004 & 3 & 2006 & Congo & - & - \\
\hline Tumbo & Male & 1989 & 18 & 1998 & Uganda & - & - \\
\hline Mika & Male & 1992 & 15 & 1998 & Uganda & - & + \\
\hline Bwambale & Male & 1999 & 7 & 2002 & Uganda & + & + \\
\hline Rutoto & Male & 2004 & 3 & 2006 & Uganda & - & + \\
\hline
\end{tabular}

Kyewunyo is captive born.

( ) sequences not analyzable.

mainly from Uganda and the Democratic Republic of Congo and a few from Rwanda (Table 1). Furthermore we provide molecular data on the SFV strains detected.

\section{MATERIALS AND METHODS}

The chimpanzee sanctuary started in 1998 and is located on Ngamba Island (S 000 06/E 32 39, $0.46 \mathrm{~km}^{2}, 1160 \mathrm{~m}$ above sea level). Chimpanzees were brought to the sanctuary after being rescued from illegal traders and poachers. The island is 100 hectares of secondary rain forest, lies $23 \mathrm{~km}$ off
Entebbe in the north-west of Lake Victoria, and is part of the Koome group of islands in the Mukono District, Uganda. The samples investigated in this study were obtained from 38 orphan chimpanzees in semi-captive management at NICS, following research approval from the relevant national authorities. The individuals tested consist of 21 females and 17 males. Table 1 summarizes the age at the time of introduction into the sanctuary and origin of the chimpanzee. Apart from the initial group of 17 chimpanzees that had lived together before being brought to the island, 
most of the chimpanzees arrived at the sanctuary at the age range from three to five years. The chimpanzees underwent a three-month quarantine period but no serological or PCR analyses were done to screen for viral pathogens.

During an annual medical health check in February, 2007, blood was collected from all chimpanzees under general anesthesia. Whole blood $(10 \mathrm{ml})$ was taken by inguinal venipuncture using CPT Vacutainer tubes. $8 \mathrm{ml}$ of blood was centrifuged at $1600 \mathrm{~g}(2800 \mathrm{rpm})$ for 30 minutes. Two aliquots of the buffy coat were refrigerated at $4^{\circ} \mathrm{C}$ for 15 minutes and then stored in cryotubes in liquid nitrogen at the Uganda Virus Research Institute till transported on dry ice to the Robert Koch-Institut in Berlin/Germany for analysis. Serum was stored at $-20^{\circ} \mathrm{C}$. The permit to export biological samples from endangered species was obtained from Uganda and German offices for the Convention on International Trade in Endangered Species (CITES).

DNA was extracted from the buffy coat using the QIAamp DNA Blood Min Kit (Qiagen, Hilden, Germany). Amplification of SFV proviral genomic region (425 bp; 6086 - 6492 on SFV reference sequence NCV001364) was performed using generic primers targeting the viral integrase (int) region as previously described [6]. Standard PCR conditions were used for both rounds of amplification with the exception of the annealing temperatures of $60^{\circ} \mathrm{C}$ for 45 cycles for the first round and $55^{\circ} \mathrm{C}$ for 45 cycles for the second round, respectively. Generic and nested PCR products were visualized in $1.5 \%$ agarose gels stained with ethidium bromide under UV light.

The purified PCR products were sequenced in both directions with PCR primers using the BigDye terminator cycle kit (Applied Biosystems, Darmstadt, Germany) on an ABI Prism 3100 automated sequencer (Applied Biosystems).

Sequences were aligned with the ClustalW program, manually edited and imported into PHYLIPversion3.67 (http://evolution.genetics.washington.edu/phylip.html). The following reference sequences were used: GenBank accession numbers were AY686179, AY583781; AY278782, X83293, AY686201, X83290, DQ354075, DQ354089, X83292; AY686203, AY686206, AY195688, AY278792, AY278776, AF049086, AY686195, AY195685, X83297, AY195682, X83296, AY639122, AY639141, AY639133, EU527588, AY639136, AY639123, AY639124, AY639138, AY639128, AY639130; EU527540, EU527532, X83294, EU527541, EU527501, EU527498, EU527637, AY195676, AY195675, EU527499, EU527496, EU527534, EU527502, X83298. Distance-based trees were generated using the Kimura two-parameter model in conjunction with the Neighbor Joining (NJ) and minimum-evolution methods in the PHYLIP software package [7]. The topology of the tree was confirmed by Maximum Likelihood analyses. The reliability of the inferred tree was evaluated by bootstrap analysis on 1000 replicates.

For serological analyses a commercially available enzyme immunoassay kit for the detection of antibodies against human T-lymphotropic virus types I and II (HTLVI/-II) was used (Murex Biotech Limited, Dartford, UK) according to the manufacturer's instructions. This test has been shown to efficiently detect STLV-1 in wild chimpanzees [5]. Simian Immunodeficiency Virus (SIV) antibody detection was performed using the Murex HIV1.2.0 kit (Abbott Murex Biotech Limited) according to the manufacturer's instructions and the GENESCREEN HIV 1.2 version 2 (BIO-RAD, München, Germany) which were designed for the detection of antibodies directed against human immunodeficiency virus and is widely used for detection of SIV in non-human primates (data will be shown elsewhere).

\section{RESULTS AND DISCUSSION}

DNA samples from 38 chimpanzees were available, 28 (73.7\%) were positive for SFV by PCR (Table $\mathbf{1}$ ). All serum samples were negative for HIV and HTLV/STLV antibodies. These results showed that none of the chimpanzees in the sanctuary was infected with SIV or STLV.

Int sequences from 23 out of 28 chimpanzee blood samples positive for SFV DNA could be used for sequence analysis. Five sequences were of poor quality due to low PCR signal and were therefore not included in this analysis. The nucleotide sequences obtained in this study were submitted to GeneBank (NCBI) and published under the accession numbers EU239510-EU239533.

Phylogenetic tree analysis was performed with the NJ method using the $23 \mathrm{SFV}$ sequences generated in this study and published relevant SFV sequences from all chimpanzee subspecies, as well as from gorilla, bonobo, orangutan, African and Asian monkeys and one sequence of an SFVinfected human [4, 6, 8-10].

The obtained sequences group fell within the SFV clade of the Eastern chimpanzee subspecies (P. t. schweinfurthii) (Fig. 1). The subspecies status of the chimpanzees studied plus their genetic diversity and association was established as $P$. t. schweinfurthii by analysis of mtDNA (data not included here). Within this clade, the 23 SFV sequences grouped in four subclusters with other recently published SFV strains from East African chimpanzee subspecies. While 22 sequences grouped in subclusters 1 to 3 , one strain obtained from the individual "Umutama" clusters with recently published sequences obtained from wild chimpanzees in subcluster 4.

SFV strains from some individuals were genetically more closely related than others. The SFV strain from "Robbie", a former alpha male of the community, forms a subcluster with SFV strains from the chimpanzees "Kyewunyo", "Connie", "Baluku", "Umugezi" and "Nagoti" with 99.5 to $100.0 \%$ nucleotide identity. "Mika", the current alpha male, has $99 \%$ to $100 \%$ nucleotide identity with "Rutoto", one of the new infant arrivals at the sanctuary, and "Becky". Also sequences of "Sunday", "Masiko" and "Ikuru" showed 99.9\% nucleotide identity. It is noteworthy to mention that "Ikuru" has been frequently bitten by the other two chimpanzees. Further investigations should show whether SFV-positive individuals are infected with more than one strain.

Sanctuary chimpanzees are usually living in a limited habitat forming a large community, which leads to a high aggression level. In contrast to the natural situation, the individuals have no alternative to keeping out of a conflict or avoiding further conflicts by staying at a distance of each 


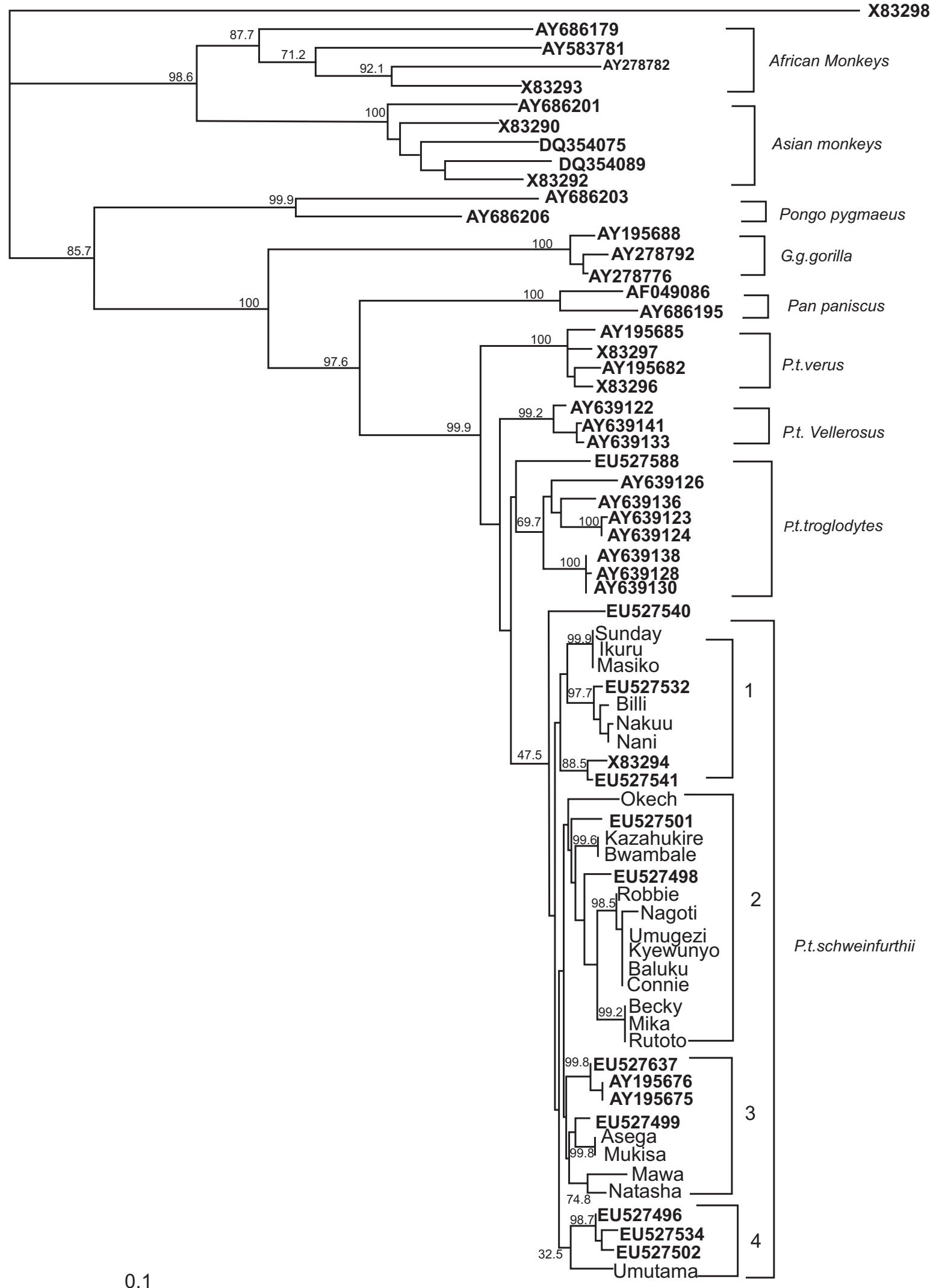

Fig. (1). Phylogenetic analysis of int sequences from SFV-infected chimpanzees of Ngamba and selected published sequences from four chimpanzee subspecies. The species' origins for the chimpanzee SFV sequences used are indicated. The phylogeny is based on the Neighbour-joining method using 2 Kimura distances performed in PHYLIP3.67 and the reliability of the inferred tree was evaluated by bootstrap analysis on 1000 replicates. The topology of the tree was confirmed by Maximum Likelihood Method. The scale bar represents an evolutionary distance of 0.1 nucleotide per site. The tree was rooted by using the new spider Monkey SFV sequence (X83298). 
other. Aggressions are mostly associated with fighting leading to scratches and also deep bite wounds. Such injuries might play an important role in transmission of pathogens $[8$, 11]. In sanctuaries this may contribute to multiple infections with strains with different genetic background. More analyses are needed to establish the frequency of infections with multiple SFV strains possibly resulting in virus recombination.

The SFV strains in this study showed a relatively high diversity and could be grouped into four main clusters. However, no clustering according to the geographic origin of the chimpanzees was found. The comparison of the strains described here with recently published SFV strains from wild $P$. t. schweinfurthii from Uganda, Congo and Tanzania [4] could also not identify clustering by geographical origin. These results are consistent with previous findings [4] which could not show region-specific clusters of SFV strains within one chimpanzee subspecies. However, this study shows in principle the possibility of pathogen introduction to great ape communities in sanctuaries which can cause co-infections with multiple virus strains.

This may have direct implications for sanctuary management since most apes are hand raised, and long-term interactions of animals and keepers with bites and scratches are frequently observed. In order to avoid pathogen transmission to humans, special precautions are needed, especially in sanctuaries where apes are SIV positive. Another concern may be that most sanctuaries aim at reintroducing great apes into the wild. The impact of these viruses on wild primates, which can again mix with regional strains, is unknown.

All wild-born captive chimpanzees on Ngamba Island were negative for SIV/HIV and STLV-1 and -2 antibodies, which might not reflect the situation in other ape sanctuaries in Africa. With the most recent findings that SIVcpz is pathogenic in free-ranging chimpanzees [1] it is obvious that all newly arriving chimpanzees at sanctuaries should be screened for defined pathogens including SIV. This will not only protect the health of the chimpanzee in the groups but also minimize the risk of infection for people in close contact with the animals. Furthermore, future reintroduction programs might benefit from such programs as only healthy or recombinant virus free animals would be released into the wild.

\section{ACKNOWLEDGEMENTS}

This research was carried out within the frame of the network "Great Ape Health Monitoring Unit" (GAHMU). The analyses were supported by Robert Koch-Institut, Berlin; a DAAD Small Research Grant; Brian Hare, MaxPlanck-Institute for Evolutionary Anthropology, Leipzig and Kim Hammond, Falls Road Hospital, Baltimore, Maryland through MGVP, International Foundation For Science (IFS). The authors thank the Chimpanzee Sanctuary and Wildlife Conservation Trust, the Uganda Wildlife Authority, the Uganda National Council of Science and Technology and CITES authorities of Uganda and Germany for permission and permits to carry out this research. Sophie Köndgen, Sabrina Neumann and Claudia Hedemann enabled the study by technical support of the investigations. The Center for Disease Control (CDC), Uganda and the Uganda Virus Research Institute provided facilities for sample storage and CDC assisted in the shipment of samples.

\section{REFERENCES}

[1] Keele BF, Jones JH, Terio KA, et al. Increased mortality and AIDS-like immunopathology in wild chimpanzees infected with SIVcpz. Nature 2009; 460: 515-9.

[2] Peters K, Barg N, Gartner K, Rethwilm A. Complex effects of foamy virus central purine-rich regions on viral replication. Virology 2008; 373: 51-60.

[3] Leendertz FH, Zirkel F, Couacy-Hymann E, et al. Interspecies transmission of simian foamy virus in a natural predator-prey system. J Virol 2008; 82: 7741-4.

[4] Liu W, Worobey M, Li Y, et al. Molecular ecology and natural history of simian foamy virus infections in wild living chimpanzees. PLoS Pathog 2008; 4: e1000097.

[5] Leendertz FH, Junglen S, Boesch C, et al. High variety of different simian T-cell Leukemia virus Type 1 strains in chimpanzees of the Taï National Park, Côte d'Ivoire. J Virol 2004; 73: 4352-6.

[6] Hussain AI, Shanmugam V, Bhullar VB, et al. Screening for simian foamy virus infection by using a combined antigen Western blot assay: evidence for a wide distribution among Old World primates and identification of four new divergent viruses. Virology 2003; 309: 248-57.

[7] Felsenstein J. PHYLIP - Phylogeny Inference Package (Version 3.2). Cladistics 1989; 5:164-6.

[8] Calattini S, Betsem EBA, Froment A, et al. Simian foamy virus transmission from apes to humans, rural Cameroon. Emerg Infect Dis 2007; 13: 1314-20.

[9] Switzer WM, Bhullar V, Shanmugam V, et al. Frequent simian foamy virus infection in persons occupationally exposed to nonhuman primates. J Virol 2004; 78: 2780-9.

[10] Wolfe ND, Switzer WM, Carr JK, et al. Naturally acquired simian retrovirus infections in central African hunters. Lancet 2004; 363: 932-7.

[11] Jones-Engel L, Engel GA, Schillaci MA, Rompis A, Putra A, Suaryana KG. Primate-to-human retroviral transmission in Asia. Emerg Infect Dis 2005; 11: 1028-35. 